\title{
Ordering ambiguity revisited via position dependent mass pseudo-momentum operators
}

\author{
Omar Mustafa ${ }^{1}$ and S.Habib Mazharimousavi ${ }^{2}$ \\ Department of Physics, Eastern Mediterranean University, \\ G Magusa, North Cyprus, Mersin 10,Turkey \\ ${ }^{1}$ E-mail: omar.mustafa@emu.edu.tr \\ ${ }^{2}$ E-mail: habib.mazhari@emu.edu.tr
}

August 9, 2018

\begin{abstract}
Ordering ambiguity associated with the von Roos position dependent mass (PDM) Hamiltonian is considered. An affine locally scaled first order differential introduced, in Eq.(9), as a PDM-pseudo-momentum operator. Upon intertwining our Hamiltonian, which is the sum of the square of this operator and the potential function, with the von Roos $d$-dimensional PDM-Hamiltonian, we observed that the so-called von Roos ambiguity parameters are strictly determined, but not necessarily unique. Our new ambiguity parameters' setting is subjected to Dutra's and Almeida's [11] reliability test and classified as good ordering.
\end{abstract}

PACS numbers: 03.65.Ge, 03.65.Fd,03.65.Ca

\section{Introduction}

Quantum mechanical Hamiltonians with position dependent mass (PDM) constitute interesting and useful models for the study of many physical problems [1-31]. They are used in the energy density many-body problem [1], in the determination of the electronic properties of the semiconductors [2] and quantum dots [3], in quantum liquids [4], in ${ }^{3} \mathrm{He}$ clusters [5] and metal clusters [6], in the Bohmian approach to quantum theory (cf., e.g. [7]), in the full and partial wave-packet revivals(cf., e.g., [8]), etc. Comprehensive reviews on the applicability of such position dependent mass settings could be found in the sample of references in [1-12]

However, it is concreted that an ordering ambiguity conflict arises in the process of defining a unique kinetic energy operator, due non-commutativity between the momentum operator $\hat{p}_{x}=-i \partial_{x}$ and the position dependent mass $M(x)=m_{\circ} m(x)$. A problem that has shown poor advancement over the last few decades. 
In general, working on PDM Hamiltonians is inspired by the von Roos Hamiltonian [15] proposal (with $\hbar=2 m_{\circ}=1$ )

$$
H=-\frac{1}{2}\left[m(x)^{\alpha} \partial_{x} m(x)^{\beta} \partial_{x} m(x)^{\gamma}+m(x)^{\gamma} \partial_{x} m(x)^{\beta} \partial_{x} m(x)^{\alpha}\right]+V(x)
$$

subjected to von Roos constraint

$$
\alpha+\beta+\gamma=-1 ; \alpha, \beta, \gamma \in \mathbb{R}
$$

Hamiltonian (1) may, in a straightforward manner and with the constraint (2), be very well recast ( cf., e.g., [10]) as

$$
H=-\partial_{x}\left(\frac{1}{m(x)}\right) \partial_{x}+\tilde{V}(x),
$$

with

$$
\tilde{V}(x)=\frac{1}{2}(1+\beta) \frac{m^{\prime \prime}(x)}{m(x)^{2}}-[\alpha(\alpha+\beta+1)+\beta+1] \frac{m^{\prime}(x)^{2}}{m(x)^{3}}+V(x),
$$

where primes denote derivatives. Obviously, nevertheless, the profile of $\tilde{V}(x)$ (namely the first two terms in (4)) changes as the parameters $\alpha, \beta$, and $\gamma$ change, manifesting therefore the eruption of ordering ambiguity in the process of choosing the kinetic energy operator $\hat{T}$. Hence, $\alpha, \beta$, and $\gamma$ are usually called the von Roos ambiguity parameters.

Several proposals for the kinetic energy operator are suggested in literature. Amongst exist; the Gora and Williams $(\beta=\gamma=0, \alpha=-1)[16]$, Ben Danial and Duke $(\alpha=\gamma=0, \beta=-1)$ [17], Zhu and Kroemer $(\alpha=\gamma=-1 / 2, \beta=0)$ [18], and Li and Kuhn $(\beta=\gamma=-1 / 2, \alpha=0)$ [19]. However, the Hermiticity of the kinetic energy operator, the current density conservation, the experimental results [20-25], and the condensed matter theories [26,27] may give some ideas on the identity of the von Roos ambiguity parameters. Applying Hamiltonian (1) to an abrupt heterojunction between two crystals (cf., e.g., sample of references in $[25-28,30]$ ), for example, implied that for $\alpha \neq \gamma$ the wave function vanishes at the heterojunction (i.e., the heterojunction plays the role of an impenetrable barrier). Hence, the only feasible cases are due $\alpha=\gamma$ to ensure the continuity of $m(x)^{\alpha} \psi(x)$ and $m(x)^{\alpha+\beta}\left[\partial_{x} \psi(x)\right]$ at the heterojunction boundary.

Very recently, however, Dutra and Almeida [11] have carried out a reliability test on the orderings available in literature. They have used an exactly solvable Morse model and concluded that the orderings of Gora and Williams $(a=\beta=$ $\gamma=0, \alpha=-1)[16]$, and Ben Danial and Duke $(a=\alpha=\gamma=0, \beta=-1)$ [17] should be discarded for they result in complex energies. Nevertheless, they have classified the ordering of Zhu and Kroemer $(a=0, \alpha=\gamma=-1 / 2, \beta=0)$ [18], and that of Li and Kuhn $(a=\alpha=0, \beta=\gamma=-1 / 2)$ [19] as good orderings. Yet, they have shown that Weyl (cf., e.g., Borges in [30]) and Li and Kuhn [19] orderings are equivalent. 
Ultimately, therefore, the continuity conditions at the heterojunction boundaries and Dutra's and Almeida's [11] reliability test single out Zhu and Kroemer $(a=0, \alpha=\gamma=-1 / 2, \beta=0)$ [18] as good ordering. This, in effect, inspires our current methodical proposal and manifests the introduction of a PDM-pseudomomentum operator which, in turn, leads to a new good ordering.

On the other hand, within a Liouvillean-type change of variables spiritual lines, the point canonical transformation (PCT) method for Schrödinger equation often mediates a transition between two different effective potentials. That is, in the PCT settings, one needs the exact solution of a potential model in a class of shape invariant potentials to form the so-called reference/old potential. The reference/old potential along with its exact solution (i.e. eigenvalues and eigenfunctions) is then mapped into the so-called target/new potential, hence exact solution for the target/new potential is obtained. For more details on this issue the reader may refer to, e.g., ref. [14].

In this parer, we recollect (in section 2) the $d$-dimensional form of the von Roos Hamiltonian suggested by Quesne [10] and introduce our PDM-pseudomomentum operator. The consequences of such operator's setting on the von Roos ambiguity parameters are given in the same section. Moreover, the corresponding $d$-dimensional radial Schrödinger Hamiltonian and the PCT $d$-dimensional mapping are also reported. Our concluding remarks are given in section 3.

\section{$2 d$-dimensional von Roos Hamiltonian and PDM- pseudo-momentum operators}

Quesne in [10] has suggested a general form of von Roos $d$-dimensional PDM Schrödinger equation

$$
\begin{gathered}
\left\{-\frac{1}{2}\left[m(\mathbf{q})^{\alpha} \partial_{j} m(\mathbf{q})^{\beta} \partial_{j} m(\mathbf{q})^{\gamma}+m(\mathbf{q})^{\gamma} \partial_{j} m(\mathbf{q})^{\beta} \partial_{j} m(\mathbf{q})^{\alpha}\right]\right\} \psi(\mathbf{q}) \\
+\{V(\mathbf{q})-E\} \psi(\mathbf{q})=0,
\end{gathered}
$$

where $\mathbf{q}=\left(q_{1}, q_{2}, \cdots, q_{d}\right), \partial_{j}=\partial / \partial q_{j}, j=1,2, \cdots, d, m(\mathbf{q})$ is the dimensionless form of the mass $M(\mathbf{q})=m_{\circ} m(\mathbf{q}), V(\mathbf{q})$ is the potential function, and summation runs over repeated indices. In this case, the $d$-dimensional PDM Schrödinger Hamiltonian reads

$$
H=-\partial_{j}\left(\frac{1}{m(\mathbf{q})}\right) \partial_{j}+\tilde{V}(\mathbf{q})
$$

with

$$
\tilde{V}(\mathbf{q})=\frac{1}{2}(1+\beta) \frac{\partial_{j} \partial_{j} m(\mathbf{q})}{m(\mathbf{q})^{2}}-[\alpha(\alpha+\beta+1)+\beta+1] \frac{\left[\partial_{j} m(\mathbf{q})\right]^{2}}{m(\mathbf{q})^{3}}+V(\mathbf{q}) .
$$

Let us now consider, for simplicity, quasi-free-particles' setting (i.e., $V(\mathbf{q})=$ 0 ). Then it would be obvious that the quasi-free-particles' Hamiltonian structure 
suggests that the kinetic energy operator

$\hat{T}=-\partial_{j}\left(\frac{1}{m(\mathbf{q})}\right) \partial_{j}+\frac{1}{2}(1+\beta) \frac{\partial_{j} \partial_{j} m(\mathbf{q})}{m(\mathbf{q})^{2}}-[\alpha(\alpha+\beta+1)+\beta+1] \frac{\left[\partial_{j} m(\mathbf{q})\right]^{2}}{m(\mathbf{q})^{3}}$

may, mathematically speaking, very well be expressed as the square of a firstorder differential vector operator of a general form

$$
\hat{\Pi}_{j}=-i\left\{F(m(\mathbf{q})) \partial_{j}+G_{j}(m(\mathbf{q}))\right\} .
$$

This would $\left(\right.$ with $F(m(\mathbf{q})) \equiv F(\mathbf{q})=F, G_{j}(m(\mathbf{q})) \equiv G_{j}(\mathbf{q})=G_{j}$ for simplicity, and $\left.\hat{T}=\hat{\Pi}^{2}=\delta_{i j} \hat{\Pi}_{i} \hat{\Pi}_{j}\right)$ imply

$$
\hat{T}=-F^{2} \partial_{j} \partial_{j}-\left[F\left(\partial_{j} F\right)+2 F G_{j}\right] \partial_{j}-\left[F\left(\partial_{j} G_{j}\right)+G_{j} G_{j}\right],
$$

If we compare Eq.(10) with (8) we obtain

$$
\begin{gathered}
F(\mathbf{q})= \pm \frac{1}{\sqrt{m(\mathbf{q})}} \\
\frac{\partial_{j} m(\mathbf{q})}{m(\mathbf{q})^{2}}=-2 F \partial_{j} F=-\left[F\left(\partial_{j} F\right)+2 F G_{j}\right] \\
G_{j}=\frac{\partial_{j} F}{2}
\end{gathered}
$$

The structure of our first-order differentioal operator is therefore clear and can be cast as

$$
\hat{\Pi}_{j}=-i\left\{F(\mathbf{q}) \partial_{j}+\frac{1}{2}\left[\partial_{j} F(\mathbf{q})\right]\right\} .
$$

At this point, it should be noted that our operator $\hat{\Pi}$ is Hermitian and represents the position-dependent-mass generalization of the ordinary momentum operator $\hat{p}_{j}=-i \partial_{j}$ (i.e., at constant mass settings $M(x)=m_{\circ}$ ). Hence, $\hat{\Pi}$ could be labeled, hereinafter, as a PDM-pseudo-momentum operator.

\subsection{Consequences of our PDM-pseudo-momentum oper- ator $\hat{\Pi}$ on the von Roos ambiguity parameters}

In a straightforward manner it is easy to show that

$$
\hat{\Pi}^{2}=-\partial_{j}\left(\frac{1}{m(\mathbf{q})}\right) \partial_{j}+\frac{1}{4} \frac{\partial_{j} \partial_{j} m(\mathbf{q})}{m(\mathbf{q})^{2}}-\frac{7}{16} \frac{\left[\partial_{j} m(\mathbf{q})\right]^{2}}{m(\mathbf{q})^{3}} .
$$

Comparing this result with the kinetic energy operator $T$ in (8) we obtain

$$
(1+\beta)=\frac{1}{2}, \quad[\alpha(\alpha+\beta+1)+\beta+1]=\frac{7}{16},
$$


which in turn suggests that the von Roos ambiguity parameters are strictly determined (but not necessarily unique) as

$$
\beta=-\frac{1}{2}, \text { and } \alpha=\gamma=-\frac{1}{4} .
$$

Hence, the $d$-dimensional von Roos Hamiltonian reads

$$
H=-\partial_{j}\left(\frac{1}{m(\mathbf{q})}\right) \partial_{j}+\tilde{V}(\mathbf{q})=-\vec{\nabla}_{d}\left(\frac{1}{m(\mathbf{q})}\right) \cdot \vec{\nabla}_{d}+\tilde{V}(\mathbf{q})
$$

with

$$
\tilde{V}(\mathbf{q})=\frac{1}{4} \frac{\partial_{j} \partial_{j} m(\mathbf{q})}{m(\mathbf{q})^{2}}-\frac{7}{16} \frac{\left[\partial_{j} m(\mathbf{q})\right]^{2}}{m(\mathbf{q})^{3}}+V(\mathbf{q}) .
$$

At this point, one may wish to subject such ambiguity parameters' settings (15) to Dutra's and Almeida's [11] reliability test on the exactly solvable onedimensional Morse model (see equations (10)-(16) in [11]). Such test shows that the ambiguous term $\nu(\alpha, \beta, \gamma, a)=\sqrt{1 / 4-2 q / c^{2}}=1 / 4$ (i.e., equation(16) in [11] for $\alpha=\gamma=-1 / 4, \beta=-1 / 2)$ and classifies our ordering as a good-ordering (along with that of Zhu's and Kroemer's [18].

\subsection{Corresponding $d$-dimensional radial Schrödinger Hamil- tonian}

We, in the forthcoming developments, shall assume the radial symmetrization of $m(\mathbf{q})$ and $V(\mathbf{q})$ in the $d$-dimensional radially symmetric Schrödinger Hamiltonian for $d \geq 2$. Under these settings, (17) and (18) imply

$$
H_{r, d}=-\partial_{j}\left(\frac{1}{m(r)}\right) \partial_{j}+\tilde{V}(r)=-\vec{\nabla}_{d}\left(\frac{1}{m(r)}\right) \cdot \vec{\nabla}_{d}+\tilde{V}(r)
$$

where

$$
\tilde{V}(r)=\frac{1}{4} \frac{\partial_{r}^{2} m(r)}{m(r)^{2}}-\frac{7}{16} \frac{\left[\partial_{r} m(r)\right]^{2}}{m(r)^{3}}+V(r) ; \quad \mathbb{R} \ni r \in(0, \infty)
$$

Recollecting that the $d$-dimensional wave function for radially symmetric Schrödinger equation is given by

$$
\Psi(\vec{r})=r^{-(d-1) / 2} R_{n_{r}, \ell_{d}}(r) Y_{\ell_{d}, m_{d}}\left(\theta_{1}, \theta_{2}, \cdots, \theta_{d-2}, \varphi\right) .
$$

would, in turn, when substituted in

$$
\left\{-\vec{\nabla}_{d}\left(\frac{1}{m(r)}\right) \cdot \vec{\nabla}_{d}+\tilde{V}(r)\right\} \Psi(\vec{r})=E_{d} \Psi(\vec{r}),
$$

results in the following $d$-dimensional radial Schrödinger equation

$$
\left\{\frac{d^{2}}{d r^{2}}-\frac{\ell_{d}\left(\ell_{d}+1\right)}{r^{2}}+\frac{m^{\prime}(r)}{m(r)}\left(\frac{d-1}{2 r}-\frac{d}{d r}\right)-m(r)\left[\tilde{V}(r)-E_{d}\right]\right\} R_{n_{r}, \ell_{d}}(r)=0 .
$$


Where $\ell_{d}=\ell+(d-3) / 2$ for $d \geq 2, \ell$ is the regular angular momentum quantum number, and $n_{r}=0,1,2, \cdots$ is the radial quantum number. Of course, equation (23) is privileged with the inter-dimensional degeneracies associated with the isomorphism between angular momentum $\ell$ and dimensionality $d$. On the other hand, moreover, the $d=1$ (with $\mathbb{R} \ni r \in(0, \infty) \longrightarrow \mathbb{R} \ni x \in(-\infty, \infty)$ ) case can be obtained through the trivial substitutions $\ell_{d}=-1$ and $\ell_{d}=0$ for even and odd parity, $\mathcal{P}=(-1)^{\ell_{d}+1}$, respectively. Yet, a unique isomorphism exists between the $S$-wave $(\ell=0)$ energy spectrum in 3D and in 1D. On this issue, the reader may wish to refer to, e.g., Mustafa and Znojil [32], and Mustafa and Mazharimousavi $[13,14,31]$ and references cited therein.

\subsection{Corresponding PCT $d$-dimensional mapping}

In this section, we closely follow Mustafa's and Mazharimousavi's recipe discussed in [14]. Where, a substitution of the form $R(r)=m(r)^{1 / 4} \phi(Z(r))$ in (22) would result in $Z^{\prime}(r)=\sqrt{m(r)}$, manifested by the requirement of a vanishing coefficient of the first-order derivative of $\phi(Z(r))$ ( hence a one-dimensional form of Schrödinger equation is achieved), and suggests the following point canonical transformation

$$
Z(r)=\int^{r} \sqrt{m(y)} d y \Longrightarrow \phi_{n_{r}, \ell_{d}}(Z(r))=m(r)^{-1 / 4} R_{n_{r}, \ell_{d}}(r) .
$$

Which in effect implies

$$
\left\{-\frac{d^{2}}{d Z^{2}}+\frac{\ell_{d}\left(\ell_{d}+1\right)}{r^{2} m(r)}+V_{e f f}(r)-E_{d}\right\} \phi_{n_{r}, \ell_{d}}(Z)=0
$$

where

$$
V_{e f f}(r)=V(r)-U_{d}(r) ; \quad U_{d}(r)=\frac{m^{\prime}(r)(d-1)}{2 r m(r)^{2}} .
$$

It should be noted, however, that the definition of $U_{d}(r)$ in $(25)$ is now more simplified than that in Eq. (8) of Mustafa and Mazharimousavi in [14].

On the other hand, an exactly solvable (including conditionally-exactly or quasi-exactly solvable) $d$-dimensional time-independent radial Schrödinger wave equation (with a constant mass $M(x)=m_{\circ}$ and $\hbar=2 m_{\circ}=1$ units)

$$
\left\{-\frac{d^{2}}{d Z^{2}}+\frac{\mathcal{L}_{d}\left(\mathcal{L}_{d}+1\right)}{Z^{2}}+V(Z)-\varepsilon\right\} \psi_{n_{r}, \ell_{d}}(Z)=0
$$

would form a reference for the exact solvability of the target equation (24). That is, if the exact/conditionally-exact/quasi-exact solution (analytical/numerical) of (26) is known one can construct the exact/conditionally-exact/quasi-exact solution of (24) through the relation

$$
\frac{\ell_{d}\left(\ell_{d}+1\right)}{r^{2} m(r)}+V(r)-U_{d}(r)-E_{d} \Longleftrightarrow \frac{\mathcal{L}_{d}\left(\mathcal{L}_{d}+1\right)}{Z^{2}}+V(Z)-\varepsilon,
$$


Where $\mathcal{L}_{d}$ is the $d$-dimensional angular momentum quantum number of the reference Schrödinger equation. The reference -target map is therefore complete and an explicit correspondence (cf. e.g., Znojil and Lévai [33] and Mustafa and Mazharimousavi $[13,14]$ ) between two bound state problems is obtained.

A power-law position dependent mass of the form $m(r)=\varsigma r^{v}$, for example, would imply a PCT function

$$
Z(r)=\sqrt{\varsigma} \int^{r} y^{v / 2} d y=\frac{2 \sqrt{\varsigma}}{(v+2)} r^{(v+2) / 2} \Longrightarrow \frac{(v+2)}{2} Z(r)=r \sqrt{m(r)}
$$

and hence equation (26) gives

$$
U_{d}(r)=\frac{v(d-1)}{2 r^{2} m(r)} \equiv \frac{2 v(d-1)}{(v+2)^{2} Z(r)^{2}} ; \quad v \neq-2
$$

Relation (28) in effect reads,

$$
\frac{\lambda(\lambda+1)}{r^{2} m(r)}\left(\frac{v}{2}+1\right)^{2}+V(r)-E_{d} \Longleftrightarrow \frac{\mathcal{L}_{d}\left(\mathcal{L}_{d}+1\right)}{Z^{2}}+V(Z)-\varepsilon,
$$

with

$$
\lambda=-\frac{1}{2}+|v+2|^{-1} \sqrt{\left(\frac{v}{2}+1\right)^{2}+4 \ell_{d}\left(\ell_{d}+1\right)+2 v(1-d)} ; \quad v \neq-2 .
$$

However, for $v=-2 \Longrightarrow m(r)=\alpha r^{-2}$ we obtain

$$
Z(r)=\sqrt{\varsigma} \int^{r} t^{-1} d t=\sqrt{\varsigma} \ln r
$$

and hence

$$
\begin{aligned}
\tilde{U}_{d}(v=-2) & =\frac{\ell_{d}\left(\ell_{d}+1\right)}{\varsigma}-U_{d}(r, v=-2) \\
& =\frac{\ell_{d}\left(\ell_{d}+1\right)+d-1}{\varsigma} .
\end{aligned}
$$

Which would only add a constant to the left-hand-side of (28) to yield, with $\mathcal{L}_{d}=0$ and/or $\mathcal{L}_{d}=-1$ (i.e., only s-states and/or $d=1$ states are available from the right-hand-side of (28)),

$$
V(r)+\tilde{U}_{d}(v=-2)-E_{d} \Longleftrightarrow V(q)-\varepsilon .
$$

\section{Concluding Remarks}

In this paper we have developed a Hermitian PDM-pseudo-momentum operator $\hat{\Pi}_{j}=-i\left\{F(\mathbf{q}) \partial_{j}+\left[\partial_{j} F(\mathbf{q})\right] / 2\right\}$, where $F(\mathbf{q})= \pm 1 / \sqrt{m(\mathbf{q})}$. Hereby, the notion of PDM-pseudo-momentum operator is inspired by the fact that $\hat{\Pi}$ has 
an in-built regular momentum operator $\hat{p}_{j}=-i \partial_{j}$, which is recoverable at constant mass settings (i.e., $M(\mathbf{q})=m_{\circ} \Longrightarrow F(\mathbf{q})= \pm 1$ ). Moreover, we have constructed our $d$-dimensional PDM-Hamiltonian, $H_{M M}=\hat{\Pi}^{2}+V(\mathbf{q})$.

On the other hand, upon intertwining our Hamiltonian, $H_{M M}$, with the von Roos $d$-dimensional PDM-Hamiltonian, $H_{v R}=\hat{T}_{d}(\alpha, \beta, \gamma)+V(\mathbf{q})$, (cf.,e.g., Quesne [10]), we have observed that the so-called von Roos ambiguity parameters (i.e., $\alpha, \beta$ and $\gamma$ ) are strictly determined (i.e., $\beta=-1 / 2$ and $\alpha=\gamma=-1 / 4$ ), but not necessarily unique of course. Therefore, the von Roos $d$-dimensional PDM-Hamiltonian collapses into

$$
H_{v R} \Longrightarrow H_{M M}=-m(\mathbf{q})^{-1 / 4} \partial_{j} m(\mathbf{q})^{-1 / 2} \partial_{j} m(\mathbf{q})^{-1 / 4}+V(\mathbf{q}) .
$$

On the logistical supportive sides of our strict determination of the von Roos ambiguity parameters $\beta=-1 / 2$ and $\alpha=\gamma=-1 / 4$, we recollect that Bagchi et al [29], while analyzing the so-called quasi-free-particle problem, have used an intertwining relationship $\eta H=H_{1} \eta$ (where $\eta$ is a Darbouxal first-order intertwining operator) and reported that such choices of the ambiguity parameters correspond to smooth mass functions $m(x)$ that signalled the formation of bound states. Yet, Koç et al. [30] have started with $\alpha=\gamma=0$ and $\beta=-1$ with constant potential $V(z)=V_{\circ}$ (equation (3) of Koc et al in [30]) in their study of transmission probabilities of the scattering problem through a square well potential with PDM barrier. However,they were forced to change the potential form (equation (4) of Koc et al in [30]) into

$$
V(z)=V_{\circ}+\frac{\hbar^{2}}{8 m(z)^{2}}\left(m^{\prime \prime}(z)-\frac{7 m^{\prime}(z)^{2}}{4 m(z)}\right)
$$

which is exactly the same form of the effective potential that comes out from our eq.(17) with the new $\beta=-1 / 2$ and $\alpha=\gamma=-1 / 4$ parameters setting (of course one should mind the units used in this paper, $\hbar=2 m_{\circ}=1$ ). Moreover, Dutra's and Almeida's [11] reliability test resulted in classifying our ordering as a good-ordering (along with that of Zhu's and Kroemer's [18], and Li's and Kuhn's [19]).

Therefore, the continuity conditions at the heterojunction boundaries and Dutra's and Almeida's [11] reliability test would ultimately single out Zhu and Kroemer $(a=0, \alpha=\gamma=-1 / 2, \beta=0)[18]$ and our new ordering $(\beta=-1 / 2$, $\alpha=\gamma=-1 / 4)$ as good orderings.

On the least consequential research stimulant side, such ambiguity parameters' setting would, in effect, flourish a production-line for new sets of exactlysolvable, quasi-exactly solvable, and conditionally-exactly solvable target/new Hamiltonian models. The point canonical transformation (PCT) method used in this work exemplifies one of the methods that generate such spectrum of exact-solvability. For example, for a reference/old exactly-solvable

$$
\tilde{V}_{e f f}(Z)=\frac{\mathcal{L}_{d}\left(\mathcal{L}_{d}+1\right)}{Z^{2}}+V(Z)
$$


in (30) there is a corresponding target/new exactly-solvable

$$
\tilde{V}_{e f f}(r)=\frac{\lambda(\lambda+1)}{r^{2} m(r)}\left(\frac{v}{2}+1\right)^{2}+V(r)
$$

where $\lambda$ is given by (31) and $v \neq-2$. Yet, a comprehensive number of illustrative examples on the generalized d-dimensional PCT is given by Mustafa and Mazharimousavi in [14]. Of course other methods designed to obtain exactsolvability do exist. Amongst, we may name the Lie algebraic method (cf., e.g., Lévai in [29], intertwining operators related to supersymmetric quantum mechanics (SUSYQM) method (cf., e.g., Quesne in [10]), and the shape-invariance technique (cf., e.g., Quesne in [10] and Cooper et al. in [29]).

On the feasible applicability side of our strictly determined von Roos ambiguity parameters, the applicability of such ambiguity parameters' recipe should not only be restricted to Hermitian PDM Hamiltonians but also to a broader class of non-Hermitian PDM $\eta$-weak-pseudo-Hermitian Hamiltonians (cf., e.g., Mustafa and Mazharimousavi [31] and related references cited therein).

Acknowledgement: We would like to thank the referee for the valuable comments and suggestions. 


\section{References}

[1] Puente A. and Casas M 1994 Comput. Mater Sci. 2441

[2] Bastard G 1988 "Wave Mechanics Applied to Semiconductor Heterostructures", Les Editions de Physique, Les Ulis

[3] Serra L I and Lipparini E 1997 Europhys. Lett. 40667

[4] Arias de Saaverda F, Boronat J, Polls A, and Fabrocini A 1994 Phys. Rev. B 504248

[5] Barranco M, Pi M, Gatica S.M., Hemandez E.S., and Navarro J. 1997 Phys. Rev. B 568997

[6] Puente A, Serra L I, and Casas M 1994 Z. Phys. D 31283

[7] Plastino A. R., Casas M and Plastino A. 2001 Phys. Lett. A281 297 (and related references therein)

[8] Schmidt A 2006 Phys. Lett. A 353459

[9] Dong S. H and Lozada-Cassou M. 2005 Phys. Lett. A 337313

Vakarchuk I.O. 2005 J. Phys. A; Math. Gen. 384727

Cai C.Y., Ren Z.Z. and Ju G.X. 2005 Commun. Theor. Phys. 431019

Roy B. and Roy P 2005 Phys. Lett. A 34070

Gonul B and Kocak M. 2005 Chin. Phys. Lett. 202742

[10] Quesne C. 2006 Ann. Phys. 3211221

Tanaka T 2006 J. Phys. A; Math. Gen. 39219

de Souza Dutra A 2006 J. Phys. A; Math. Gen. 39203

[11] de Souza Dutra A and Almeida C. A. S.2000 Phys Lett. A 27525

[12] Bagchi B, Banerjee A. Quesne C. and Tkachuk V.M. 2005 J. Phys. A; Math. Gen. 382929

Yu J. and Dong S. H. 2004 Phys. Lett. A 325194

Dekar L, Chetouani L and Hammann T.F. 1998 J. Math. Phys. 392551

[13] Quesne C. and Tkachuk V.M. 2004 J. Phys. A: Math. Gen. 374267

Jiang L. Yi L.-Z., and Jia C.-S. 2005 Phys. Lett. A 345279

Mustafa O. and Mazharimousavi S.H. 2006 Phys. Lett. A 358259 (arXive: quant-ph/0603134)

Alhaidari A.D. 2003 Int. J. Theor. Phys. 422999 
[14] Alhaidari A.D. 2002 Phys. Rev. A 66042116

Mustafa O. and Mazharimousavi S.H. 2006 J. Phys. A: Math. Gen. 39 10537 (arXive: math-ph/0602044)

[15] von Roos O 1983 Phys. Rev. B 277547

[16] Gora T. and Williams F. 1969 Phys. Rev. 177, 1179.

[17] Ben Danial D. J. and Duke C. B. 1966 Phys. Rev. 152683

[18] Zhu Q.-G. and Kroemer H. 1983 Phys. Rev. B 273519.

[19] Li T. and Kuhn K. J. 1993 Phys. Rev. B 4712760.

[20] Hagston W. E., Harrison P., Piorek T., and Stirner S.1994 Superlatt. Microstruct. 15199.

[21] Csavinszky P. and Elabsy A. M. 1988 Semicond. Sci. Technol. 31010.

[22] Galbraith I. and Duggan G. 1988 Phys. Rev. B 3810057.

[23] Brezini A., Sebbani M. and Marouf S. 1995 Phys. Status Solidi B 189389.

[24] Morrow R. A. and Browstein K. R. 1984 Phys. Rev. B 30678.

[25] Einevoll G. T. and Hemmer P. C. 1988 J. Phys. C: Solid State Phys. 21 L1193.

[26] Burt M. G. 1992 J. Phys. Condens. Matter 46651.

[27] Geller M. R. and Kohn W. 1993 Phys. Rev. Lett. 703103.

[28] Einevoll G. T. 1990 Phys. Rev. B 423497

[29] Bagchi B., Gorain P., Quesne C. and Roychoudhury R. 2004 Mod. Phys. Lett. A 192765

Lévai G 1994 J. Phys. A: Math. Gen. 273809

Cooper F., Khare A. and Sukhatme U. 1995 Phys. Rep. 251267

[30] Borges et al 1988 Phys. Rev. A 383101

Koç R., Şahinoĝlu G. and Koca M. 2005, Eur. Phys. J. B 48583 (arXive: quant-ph/0510172)

[31] Mustafa O. and Mazharimousavi S.H. 2006 Czech. J. Phys 56297 (arXive: quant-ph/0603272)

Mustafa O. and Mazharimousavi S.H. 2006 Phys. Lett. A 357295 (arXive: quant-ph/0604106)

Mustafa O. and Mazharimousavi S.H. 2006 (arXive: quant-ph/0607030)

Mustafa O. and Mazharimousavi S.H. 2006 (arXive: hep-th/0601017)

[32] Mustafa O. and Znojil M. 2002 J. Phys. A: Math. Gen. 358929

[33] Znojil M and Lévai G 2000 Phys. Lett. A 271327 